\title{
Conciencia corporal en el proceso de la formación profesional
}

\author{
Maria Evelina Gastulo Morante, ${ }^{1, a, b}$ José Luis Cervera Santiago., ${ }^{2, c, d}$
}

Gastulo MME, Cervera SJL. Conciencia corporal en el proceso de la formación profesional. Cuid salud, ene-jun 2017; 3(1):296-302.

\section{RESUMEN}

La conciencia corporal es el proceso dinámico e interactivo por el que se perciben los estados, procesos y acciones que suceden en el cuerpo, tanto a nivel interoceptivo, como propioceptivo y que pueden ser percibidos por uno mismo. Bajo este marco, la enseñanza-aprendizaje debe promover el encuentro interpersonal entre docente-estudiante, concibiéndose ambos como totalidades; de ahí la relevancia de desarrollar la conciencia corporal fundamentado en el dominio personal, cognitivo y emocional para poder impulsar el aprendizaje. Este ensayo invita trascender el rol academicista del docente para constituirse en agente transformacional con y desde su cuerpo para el aprendizaje.

Palabras clave: conciencia, relaciones interpersonales, aprendizaje, educación basada en competencias (Fuente: DeCS BIREME).

\section{INTRODUCCIÓN}

La humanidad del docente fuente de sabiduría y puente en el proceso de aprender de sus estudiantes. José Luis Cervera Santiago.

El nuevo paradigma educativo en educación superior (EES) enfatiza el aprendizaje en términos de adquisición de competencias, como saber conocer, saber hacer, saber ser y saber estar. Sin embargo, la educación superior ha venido priorizando el saber conocer y el saber hacer, valiéndose del manejo de técnicas y de la práctica de procedimientos.

Cantillo $^{1}$ señala que diversos estudios sobre la comunicación no verbal en la docencia superior han encontrado dificultades para investigar este componente
Gastulo MME, Cervera SJL. Body awareness in the process of vocational training. Cuid salud, ene-jun 2017; 3(1):296-302.

\begin{abstract}
Body awareness is the dynamic and interactive process by which the states, processes and actions that occur in the body, both at the interceptive and proprioceptive levels, can be perceived by oneself. Under this framework, teaching-learning should promote the interpersonal encounter between teacher-student, conceiving of both as totalities; Hence the relevance of developing body awareness, based on personal, cognitive and emotional mastery in order to boost learning. This essay invites to transcend the academics role of the teacher to become a transformational agent with and from his body for learning.
\end{abstract}

Keywords: Conscience, interpersonal relations, learning, competence-based education (Source: MeSH NLM).

relacional, sobre todo, por los escasos instrumentos que existen para evaluarlos desde el paradigma cuantitativo. De allí el interés de enfocar la atención en la conciencia corporal y en los componentes no verbales de la comunicación como la proxémica (espacio y distancia), kinésica (gestos y movimiento) y paralingüístico (voz, volumen, tono, timbre), que enriquecen el proceso de enseñar y aprender.

La enseñanza superior del siglo XXI esta avocada a conocer qué y cómo aprende y obtiene resultados o construye su conocimiento el estudiante; del mismo modo, cómo transfiere o los asocia con su persona y la realidad en la que se desenvuelve. Siendo vital en este proceso de enseñanza-aprendizaje el encuentro interpersonal docente-estudiante, enriquecido por la

1 Escuela de Enfermería Padre Luis Tezza afiliada a la Universidad Ricardo Palma, Lima-Perú.

2 Universidad Nacional Federico Villarreal, Lima-Perú.

a Licenciada en Educación.

b Magister en Ciencia de la Educación, con mención en Docencia Universitaria.

c Licenciado en Psicología.

d Especialista: Trainer en Programación Neurolingüística. Facilitador de grupos con Análisis Bioenergético 
comunicación verbal y no verbal, secundada por métodos, herramientas y estrategias que mejoran el logro académico y la formación humana o el crecimiento personal. Proceso que convoca a conocer la didáctica y la comunicación no verbal, para buscar soluciones a los problemas prácticos que afectan el proceso enseñanza-aprendizaje y el desarrollo personal y profesional del docente. ${ }^{2}$

De este modo, el docente y su persona se convierten en agente de motivación y desafío en el aprendizaje del estudiante, secundado por el diseño de actividades formativas para el desarrollo de las competencias respectivas, que requieren desenvolver habilidades y destrezas de comunicación intra e interpersonales, de la inteligencia corporal o cinestésica, emocional y el dominio personal; integrando el cuerpo, las emociones y los componentes mentales (inteligencia, pensamiento, lenguaje, juicio moral, imaginación, creatividad e intuición, entre otros). Si bien el cuerpo fue concebido por mucho tiempo como estructura biológica; hoy, después de muchas investigaciones, en especial de la neurociencia, se reconoce que el cuerpo posee inteligencia, memoria, sabiduría y, sobre todo, se constituye en puente para la comunicación y el aprendizaje, como refiere Casanovas $^{3}$ [...] el cuerpo es la materialización y a la vez, el medio o vehículo para pensar y sentir.

El docente universitario de hoy está llamado a trascender sus capacidades intelectivas, el manejo de métodos y técnicas psicopedagógicas y de conocimientos; dado que el instrumento de trabajo como docente, por excelencia, es su propia persona y experiencia. Así lo exige el aprendizaje por competencias y, para tal propósito, la integridad personal es una prioridad, conjugando lo biológicocorporal, lo emocional, lo mental, el dominio del yo, lo ético-moral y lo espiritual; perspectiva desde la cual la vida y la enseñanza cobra una nueva dimensión. ${ }^{4}$

Por lo tanto, el docente necesita disponer de espacios y dominar actividades orientadas a cultivar el dominio personal, desarrollar la inteligencia interpersonal; para lo cual será necesario recuperar la sensibilidad y desarrollar la conciencia corporal y la comunicación interna, viviendo así cada experiencia desde y con sentido, como lo señala Gendlin ${ }^{5}$ [...]cultivar la experiencia sentida le da soporte a la mente consciente, ya que la fuente de origen en la creación del significado procede desde niveles pre lógicos (la sensación sentida) hasta lograr una conceptualización del proceso.

Zabalza, ${ }^{6}$ basándose en sus estudios, señala que las competencias y habilidades sociales y emocionales del docente universitario son piezas claves para el encuentro docente-estudiante. Habilidades que se potencian si se desarrolla la autopercepción, la sensibilidad corporal y la comunicación intrapersonal; de allí la necesidad de cultivar, mediante técnicas psicológicas creativas y experienciales, la conciencia corporal y el dominio personal desde la perspectiva mente-cuerpo-emoción.

En concordancia con Siegel, ${ }^{7}$ la construcción de las primeras relaciones en la infancia y la niñez son la base para la construcción de las relaciones interpersonales futuras; relación interpersonal que se sustenta en los procesos internos construidos en las primeras etapas del desarrollo con las figuras significativas en las que están integradas cerebro-mente, apego y cuerpo; a partir del cual se podrán construir a futuro encuentros interpersonales saludables y creativos. Es por ello que la relación docente-estudiante es la que sostiene el aprendizaje transformacional, además de los procesos emocionales, la creatividad, la imaginación y los procesos cognitivos.

Bajo este contexto, este ensayo pone sobre el tapete que, tanto el que aprende, como el que facilita el aprendizaje, ponen en juego sus maestrías (personalidad, vocación, experiencia y conocimientos) para hacer realidad el aprendizaje por competencias, el saber conocer, hacer, ser y estar; maestrías que tienen su soporte en la integridad, la conciencia corporal, emocional y el dominio cognitivo, haciendo que el proceso de aprendizaje y el aprender recobre naturalidad, espontaneidad.

\section{La conciencia corporal}

La conciencia (awareness) implica una mayor presencia de mi facultad de ser-que-conocer. Según Merrell-Wolff, citado por Wrycza, ${ }^{8}$ el término se inclina más en la dirección de tener conocimiento de algo; además refiere que la diferencia esencial entre consciencia -con-un-contenido y conciencia sin-objeto está relacionada con el raciocinio y el pensamiento lógico de la primera y la conciencia pura/intuitiva de la segunda. La conciencia está implícita en todas las experiencias; por lo tanto, es el último requisito previo para llevar una vida mejor, una vida de calidad. Siendo así, la conciencia posee una base biológica y también afectiva (pre lógico), como lo afirma Wrycza $^{8}$, el término conciencia es la sencilla capacidad que todos tienen para reconocer la experiencia.

De este modo, la conciencia está ligada a todo aquello que se percibe; siendo que en los momentos álgidos de conciencia no solo se detecta aquello que está ocurriendo dentro del campo de la experiencia, sino que también existe conciencia de la percepción de este proceso, que podría denominarse como una autoconciencia de la conciencia. En tal sentido, Cebolla et al. ${ }^{9}$ refieren que la conciencia corporal es el proceso dinámico e interactivo por el que se perciben los estados, procesos y acciones que suceden en el cuerpo, tanto a nivel interoceptivo (capacidad para percibir con 
los sentidos los estímulos del exterior), como propioceptivo (capacidad para percibir, sentir e identificar y procesar las señales internas que trasmite el propio cuerpo) y que pueden ser percibidas por uno mismo.

Sin embargo, dadas las múltiples actividades en las que está inmerso el docente universitario, su atención y concentración termina por enfocarse en el mundo exterior, es decir, atrapado por las actividades que realiza, se distancia de a pocos de los procesos internos, de su cuerpo y de sus emociones, que finalmente terminan cerrando las puertas de acceso a la conciencia propioceptiva, volviéndose cada vez más inconsciente de la captación de las señales internas como las fisiológicas, las sensaciones y movimientos. Postura que da pie a la incubación del estrés emocional y la ansiedad, asociados a problemas cardiovasculares, problemas respiratorios, digestivos, e incluso, desencadenar enfermedades psicosomáticas por efecto de la carga emocional toxica acumulada. ${ }^{10}$

Cuando el docente universitario utiliza su inteligencia como el único recurso para su trabajo, termina desconectándose de la sabiduría de su cuerpo y de sus emociones y, a su vez, obviando información valiosa que le brinda el cuerpo a través de movimientos, sensaciones, tensiones, malestar y debilitamiento; es decir, va perdiendo la capacidad de sentir, y con ello, pierde la espontaneidad y capacidad para disfrutar de lo que realiza, sobre todo, porque termina mecanizando sus encuentros interpersonales. ${ }^{11}$

Sutton y Wheatley, citado por Rodríguez-Jimenez et al., ${ }^{12}$ señalan que [...] para lograr procesos de enseñanza-aprendizaje efectivos y de calidad, los docentes deben desarrollar las competencias emocionales; sin embargo, el logro de las competencias emocionales (habilidades de la inteligencia emocional) requieren de la conciencia corporal. $\mathrm{Al}$ respecto, Baniel $^{13}$ refiere que [...] el movimiento consciente abrirá las puertas al cambio y al crecimiento en su forma de pensar y de sentir. De este modo, el cuerpo llegaría a convertirse en el intermediario, en el puente para la comunicación interna y externa en el docente, haciendo mucho más efectiva sus relaciones con los estudiantes.

Entendido así, el cuerpo es el intermediario del aprendizaje transformacional que enriquece el encuentro interpersonal y la comunicación en el nuevo escenario de competencias del docente universitario. Zabalza ${ }^{6}$ menciona que existe una resonancia empática entre docente y estudiante, reconociendo que la enseñanzaaprendizaje es un ejercicio cuerpo a cuerpo del docente con el estudiante, que trasciende la palabra y se fortalece de la comunicación no verbal; es decir, que la mirada, la sonrisa y la postura corporal del docente dan vida al encuentro y hace presencia para el aprendizaje del estudiante.

Este diálogo cuerpo a cuerpo se sostiene en la conciencia corporal y emocional, permitiéndole al docente poseer la sensibilidad para percibir su realidad interna y la del entorno, es decir, identificar y sintonizar con los patrones interoceptivos y propioceptivos, captando el flujo energético que se traduce en el movimiento consciente, que intensifica la resonancia afectiva, la creatividad y la espontaneidad, sobre todo, es estar más atento para detectar con precisión lo que trae consigo el estudiante. ${ }^{11}$

Darle espacio al desarrollo de la conciencia corporal reivindica la humanidad del proceso enseñanza -aprendizaje; sin embargo, las capacitaciones orientadas a la formación de las competencias docentes terminan priorizando el desarrollo de habilidades para producir y no para el mejoramiento del disfrute del aprender y del hacer. Para tal, sería necesario el desarrollo de la autoconciencia, con la que podrá reconocer los estados mentales, emocionales y sentimentales y "ver" qué filtros lo están limitando, como las creencias o los pensamientos automáticos distorsionados. ${ }^{14}$

\section{El pasado, puente para el cambio}

Con el paso de los años y con el apoyo de las teorías cognitivas se dio más énfasis a los procesos cognitivos, en especial a la inteligencia como única herramienta para el aprendizaje, ignorando que la inteligencia no es exclusiva para las matemáticas y las letras, sino que es diversa (visual, sonidos, cenestésica), dinámica y única. ${ }^{15}$

Por otro lado, las actividades de formación humana para docentes y estudiantes fueron sustituidas por teorías, conceptos, metodologías y técnicas educativas que tenían como objetivo obtener un resultado, relegando el proceso y la persona que aprende; estilo de aprendizaje que también se extendió a la educación superior. Asimismo, la tecnología, en especial la cibernética, comenzó a ocupar más espacio en el escenario educativo, acentuando aún más el distanciamiento de la persona y el encuentro entre profesor y estudiante. Indicador que se contrapone a lo dicho por la Universidad Católica Silva Hernández de Santiago de Chile-Chile, donde se señala que "condicionando nuestras mentes y cuerpos correctamente es posible sacar ventaja del potencial del cerebro para la plasticidad y facilitar el proceso de aprendizaje. ${ }^{16}$

De lo descrito en el párrafo anterior, surge la necesidad de recuperar la humanidad del docente y del estudiante, enfatizando el autocuidado, la integridad, el bienestar físico y psicológico; por lo que el proceso de enseñar y aprender es por naturaleza un acto natural, secundado por entornos favorables a nivel físico-natural 
y psicosocial. De ahí la necesidad de desarrollar programas de capacitación de tipo vivencial, enfocados a desarrollar habilidades y destrezas socioemocionales sostenibles, en la que el cuerpo, como espacio vivencial, es la fuente primaria de comunicación no verbal. Si todo estimulo o evento externo o interno pasa por el cuerpo, la atención y conciencia del docente deberá estar enfocada en las actividades que realiza en la sesión de clase y teniendo como intermediario su cuerpo, y con ello aprender a relajarse, soltarse (ceder) y fluir (dejarnos tocar y envolver por el), estado donde el cuerpo encuentra su reposo natural que sabe a recuperación y bienestar. ${ }^{17}$

\section{De la conciencia corporal a la inteligencia kinestésica}

Para hablar de conciencia corporal es necesario saber que la noción de cuerpo fue estudiada por la medicina, la filosofía, la literatura, el arte y la psicología; de ahí que en los últimos 20 años las investigaciones de la neurociencia, las terapias psicocorporales que trabajan con y desde el cuerpo y, la atención plena o mindfulness, resaltan la importancia del cuerpo como instancia que trasciende lo biológico, para dar espacio a lo social, lo psicológico y lo espiritual; es decir, se hace puente para el procesamiento de los eventos internos y externos. En tal sentido, los profesionales que trabajan con personas necesitan "prestar atención de manera intencional, experiencial, sostenida y sin juzgar al momento presente" [...] “observar sin juzgar”, "sentir”. 18

El cuerpo, al ser concebido como instancia psicológica, social y espiritual, se convierte en el vehículo para el desarrollo de vínculos afectivos, teniendo su punto de partida en las primeras etapas del desarrollo humano, considerando que lo afectivo y lo corporal son una unidad y se constituyen en la base para el desarrollo de la personalidad, las relaciones interpersonales, la sexualidad y el crecimiento espiritual. ${ }^{19}$ Alemay y García ${ }^{20}$ refieren que el cuerpo se vuelve en intermediario a través del cual la persona revela su historia biográfica, y le da consistencia a los componentes afectivos, culturales, sociales $y$ espirituales, haciendo real su presencia en el entorno y en las relaciones.

Desde la perspectiva de Alemay y García, ${ }^{20}$ el cuerpo da señales de cómo funciona e interactúa con el entorno, de cómo atiende las necesidades, vive el placer y el dolor; el cuerpo se vuelve el vehículo de la vida y se hace imprescindible para el autoconocimiento por ser la memoria biográfica, permite afirmar el yo personal y explorar el mundo en general. $\mathrm{Si}$ se desea vivir creativamente y esa es la tarea, es esencial volver a él, habitarlo y vivenciarlo, es decir, prestarle atención, empezar a entender su lenguaje, conectar con su sabiduría orgásmica, escuchar sus deseos o acompañar su cansancio y su dolor en un contínuo y sorpresivo proceso de aprendizaje. ${ }^{20}$
Desde esta visión el cuerpo pasó a ser un cuerpo real, concebido como una compleja red de interrelaciones, donde intervienen el sistema biológico, psicológico, somático, histórico y social del sujeto, que dan cuenta de su existencia entendida como realidad global; perspectiva que la sociedad y quienes trabajan con personas perciben el cuerpo como estructura, para así desarrollar actividades hacia el exterior, usándolo como instrumento, como herramienta de trabajo; mas no como fuente de vida, de socialización, de encuentro y de afirmación de la identidad personal. Sin embargo, el cuerpo se convirtió en depositario de la carga emocional tóxica como la cólera, ira, resentimiento, miedo y tristeza; emociones que al ser sofocadas y sepultadas se transforman en agotamiento, cansancio; dando origen al estrés y la ansiedad. ${ }^{21}$

Al haber recuperado el cuerpo la categoría de organismo vivo, compuesto de la energía que lo impulsa a interactuar creativamente con el entorno y con el ambiente natural; esta interacción le permite entrar en contacto con eventos cargados de energía positiva, permitiéndole potenciar y mejorar el flujo energético, que no solo trasciende su vitalidad como organismo vivo, sino que enriquece el lenguaje no verbal y por consiguiente, mejora la comunicación. Cuando el cuerpo está cargado de eventos con energía negativa se descompensa y aparece la mala vibra, que inhibe y restringe el movimiento y la expresión corporal. ${ }^{22}$

Por ejemplo, cuando un docente se dirige al salón de clase alegre y optimista, pero al entrar en contacto con los estudiantes -su cuerpo con su sensibilidad percibe más rápido que la mente racional- percibe una carga negativa en el ambiente, esta señal le serviría como alarma para que busque una salida creativa; más como carece de recursos de manejo y regulación emocional, al poco tiempo cambia de estado de ánimo y no sabe por qué ni cómo ocurrió; mecanismo que termina generándole mal humor, dolor de cabeza y/o malestar corporal.

El cuerpo es más que una estructura físicobiológica, posee energía y sabiduría natural que no solamente contribuye a la supervivencia y la adaptación, sino que se convierte en un medio para el aprendizaje, el desarrollo de conductas creativas $\mathrm{y}$, sobre todo, al conjugarse con las experiencias gratificantes del entorno se convierte en puente para el bienestar psicológico y espiritual tal y como lo señala Lowen. ${ }^{21}$

El lenguaje del cuerpo recobra vida en el aprendizaje por competencias cuando se percibe a la persona holísticamente, pues tanto el que aprende como el que enseña, no pueden quedarse anclados en la inteligencia lógico racional, sino que deben crear puentes para desarrollar otras inteligencias como el kinestésico. $^{23} \mathrm{La}$ inteligencia corporal cobra vida, cuando la espontaneidad y la conciencia se conjugan 
para ser cualidades esenciales del movimiento corporal que conforman la expresividad motriz adecuada y óptima para el contexto, componentes necesarios para la comunicación grupal. Al desarrollar la inteligencia kinestésica, el movimiento se expresa en creatividad, de ahí la necesidad del docente recuperar la espontaneidad, la expresividad y vivir conscientemente, vivir el presente; es decir, estar en sintonía con el sentir, el hacer y el pensar. ${ }^{17}$

\section{Conciencia corporal puente para el encuentro docente-estudiante}

La conciencia corporal es un proceso dinámico que se expresa en vibraciones y movimientos, que demanda la presencia de un conductor capaz de leer el lenguaje del movimiento, además de haber aprendido a enfocar la atención en las señales internas del cuerpo; cuando se bloquea o se obstruye el flujo se inhibe el movimiento, quitándole expresividad y vitalidad a la comunicación. Al liberarse las tensiones y las cargas emocionales depositadas en partes específicas del cuerpo se recupera el flujo y, consecuentemente, el movimiento y la expresividad, la conexión y el flujo sináptico entre la zona pre frontal y el sistema límbico. Por lo tanto, el foco de atención y concentración repercute en el aprender y en el desempeño académico. De ese modo, la persona no solo mejora el campo perceptivo, se siente vivo en su entorno, mejora la percepción que tiene de sí mismo y con el entorno, sintiéndose competitivo y abierto a vivir nuevas experiencias. ${ }^{24}$

En el ser humano la capacidad propioceptiva e interoceptiva es producto del desarrollo de la conciencia biológico-corporal que le permite mejorar su adaptación, el desarrollo de su personalidad, lo que enriquece sus relaciones interpersonales, convivencia y bienestar psicológico. Proceso que finalmente impulsa a promover la educación somática o corporal en las instituciones educativas y universitarias, a fin de mejorar la calidad de vida dentro del contexto de la sociedad moderna y tecnológica. ${ }^{25}$

Al concebir el cuerpo más allá de lo puramente biológico, éste se convierte en un lugar donde ocurren las experiencias, donde se observa cómo funciona lo psíquico, lo mental y la interacción con el entorno. De ahí que las experiencias interpersonales no solo activan nuestro cuerpo, sino que también, activan y regulan la actividad mental que es producto de la actividad cerebral, y tiene su base en las primeras etapas del ciclo evolutivo. ${ }^{7}$

Según Ramos, ${ }^{26}$ practicar la respiración consciente por espacio de 10-15 minutos diarios permite enfocar la atención y concentración en este acto, extendiéndose a las actividades que se realizan en la vida cotidiana; por otro lado, ayuda a desarrollar destrezas para dialogar con las señales de malestar, incomodidad, emociones o pensamientos automáticos que repercuten en el cuerpo y activan emociones. Lo cual sería importante para saber controlar las emociones y pensamientos con carga energética negativa, ayudando a promover una sensación de bienestar y crecimiento humano.

Así siendo, la conciencia corporal demanda como primer paso volver hacia uno mismo, a la casa personal, empezando por el propio cuerpo, asumiendo una actitud positiva con la vida $\mathrm{y}$, de ese modo, atreverse a desarrollar las destrezas de saber observar, aprender a enfocar la atención y concentración hacia el interior, haciéndose presente y estando listos para acoger las señales que el cuerpo comunica.

\section{CONSIDERACIONES FINALES}

El proceso de enseñanza-aprendizaje de nivel superior en el tercer milenio insta al docente a sensibilizarse y hacer presencia interactiva con el estudiante, trascendiendo la formación técnica e informativa y recuperando la humanidad en esta relación. Desafío que reclama la humanidad del docente, al estar en sintonía consigo mismo y en comunicación armoniosa con las dimensiones de su personalidad: cuerpo, emociones, pensamientos y su yo personal. Desde esta humanidad integrada podrá ayudar al estudiante a crear puentes, no solo para conocer y manejar conceptos, desarrollar habilidades procedimentales para solucionar los problemas relacionados con la carrera en estudio; sino por el contario, enfocar la atención en la formación humana, desarrollando las habilidades y destrezas de comunicación intra e interpersonal y el dominio personal.

De este modo, la conciencia corporal se constituye en puente entre el mundo interno y externo del docente y del estudiante, participando ambos activamente en el aprendizaje; para tal proceso ellos deberán estar despiertos y en atención plena frente a lo que asumen como emociones, pensamientos y deseos- y lo que realizan.

En tal sentido, la creación de espacios y de eventos orientados a la formación humana del docente se convierten en una prioridad, motivándolos a reflexionar acerca de la importancia de la inteligencia corporal, afectivo-emocional y del dominio personal; componentes básicos para que pueda autoconocerse e interactuar creativamente con su entorno, dejando fluir energías positivas en el proceso del aprendizaje y fortalecimiento de las funciones cognitivas necesarias para la adaptación y bienestar psicológico de los estudiantes. La conciencia corporal y afectivoemocional en sincronía con lo cognitivo contribuyen en el proceso de aprender, en el desarrollo de comportamientos creativos orientados al logro de metas, la convivencia y la adaptación. El adecuado uso de herramientas naturales como la respiración consciente y el silencio interior son impostergables, además de 
cultivar hábitos saludables relacionados con el autocuidado, el afecto corporal y el bienestar psicológico.

Estas reflexiones de humanizar la enseñanza aprendizaje -devuelta a la casa personal- se inician utilizando como instrumento al propio cuerpo, siendo necesario capacitar a los docentes con programas vivenciales y así poder desarrollar la cultura somática y afectiva, es decir, educar para la vida, tomando como punto de partida la sensibilidad afectiva y la conciencia corporal, el fortalecimiento del proceso relacional y procedimental, solo de esta forma los docentes y

\section{Correspondencia:}

Maria Evelina Gastulo Morante

Correo electrónico: maria.gastulo@correo.eeplt.edu.pe

\section{REFERENCIAS BIBLIOGRÁFICAS}

1. Cantillo SMG. El uso del lenguaje no verbal en la comunicación docente universitaria, implicaciones y efectos en la eficacia comunicativa [tesis doctoral]. [internet]. Alicante-España: Universidad de Alicante; 2014 [citado 10 mar 2017]. p. 85. Disponible en: https://rua.ua.es/dspace/bitstream/10045/46195/1/tes is martha graciela cantillo sanabria.pdf

2. Amar RVM. Didáctica y comunicación no verbal. Comunicación Social. Salamanca-España: Ediciones y Publicaciones; 2014.

3. Casanovas L. La memoria corporal. $2^{\mathrm{a}}$ ed. España:Desclee de Brouwer; 2003.

4. Gonzales PC. Maestros de corazón. Un salto cuántico en la enseñanza. España. Editorial Desclee de Brouwer; 2013.

5. Gendlin E. Focusing. Proceso y técnica del enfoque corporal. $5^{\mathrm{a}}$ ed.España: Ediciones Mensajero; 2010.

6. Zabalza MA. Competencias docentes del docente universitario. Calidad y desarrollo profesional. Madrid: Narcea; 2006. p. 180, 181.

7. Siegel DJ. La mente en desarrollo. Cómo interactúan las relaciones y el cerebro para modelar nuestro ser. España: Desclee de Brouwer; 2007. p. 449.

8. Wrycza P. PNL Darse cuenta. El desarrollo de la conciencia y la percepción. Madrid España: Gaia Ediciones; 1999. p. 10, 24.

9. Cebolla A, García-Campayo T, Demarzo M. Mindfulness y ciencia de la tradición a la modernidad. España: Alianza Editorial; 2014. p. 81.

10. Lowen A.Autobiografia. Honrar al cuerpo. España: Editorial Sirio; 2004. p. 275-286. estudiantes vivirán en presencia el proceso enseñanzaaprendizaje.

\section{Declaración de financiamiento y de conflictos de interés:}

Este ensayo fue financiado por los autores, quienes declaran no tener conflictos de interés.

\section{Contribución de auditoría:}

Gastulo MME y Cervera SJL: Concepción, redacción, análisis e interpretación de la información y aprobación de la versión que será publicada.

11. Weiner CA. Focusing en la práctica clínica. La esencia del cambio. España: Editorial Desclee de Brouwer; 2016. p. 112.

12. Rodríguez-Jiménez RM, Velasco-Quintana PJ, Terrón-López MJ. Construyendo Universidades Saludables: conciencia corporal y bienestar personal. RIE [revista de internet]. 2014 [citado 10 feb 2017]; 66: 207-223. Disponible en: https://dialnet.unirioja.es/servlet/articulo?codigo $=49$ $\underline{78206}$

13. Baniel A. Movimiento consciente. Despertar de la mente para recupera la vitalidad. España: Urano; 2009. p. 58-59.

14. Cañizares OCGL (Coord). Hazte experto en Inteligencia emocional. España: Desclee de Brouwer; 2014. p. 168.

15. Mahony Terry. El poder de las Palabras. España: Desclee de Brouwer; 2009. p. 78.

16. Universidad Católica Silva Henríquez. La Comprensión del Cerebro. El nacimiento de una ciencia del aprendizaje. Santiago de Chile-Chile: UCSH, Organización para la Cooperación y el Desarrollo Económico; 2009. p.424.

17. Lopez GL. El maestro atento. Gestión consciente del aula. España: Desclee de Brouwer; 2017. p.63, 103.

18. Kabat-Zinn J. La práctica de la atención plena. España: Kairos; 2007. p. 115.

19. Wallin DJ. El apego en psicoterapia. Bilbao-España: Desclee de Brouwer, 2012. p. 24.

20. Alemany C, Garcìa V. El cuerpo vivenciado y analizado. España: Desclèe de Brouwer SA, 1996. p. 25 . 
21. Lowen A. La voz del cuerpo. El Papel del cuerpo en psicoterapia. España: Sirio; 2005. p. 221.

22. Lowen A. La Bioenergética. México: Diana; 2005. p. 41.

23. Gardner H. La estructura de la mente. La Teoría de Las Inteligencias Múltiples. Colombia: Fondo de Cultura Económica Ltda. 6ta reimpresión, 2001.p. 165-187.

24. Rodríguez JRM, Caja LMM, Parra PG, Velasco QPJ, TerrónLMJ. Inteligencia emocional y comunicación: la conciencia corporal como recurso. REDU [serie de internet]. Ene-abr 2013 [citado 15 ene 2017];11 (1): 213-241. Disponible en:
https://polipapers.upv.es/index.php/REDU/article/vi ew/5598/5589

25. Somatic Systems Institute. A conversation with Thomas Hanna PhD by Helmut Milz, MD [internet].[S.1]: Somatic Systems Institute; [s.f.]. [actualizado s.f.; citado 10 dic 2016]. [cerca 03 laudas]. Disponible en: https://somatics.org/library/biblio/mhhannaconversation

26. Ramos DN, Enríquez H, Recondo O. Inteligencia emocional plena. Mindfulness y la gestión eficaz de las emociones. España: Kairos; 2012. p. 112, 127. 